\title{
Modulation Methods for Neutral-Point-Clamped Wind Power Converter Achieving Loss and Thermal Redistribution under Low- Voltage-Ride-Through
}

\author{
Ke Ma, Member, IEEE and Frede Blaabjerg, Fellow, IEEE
}

\begin{abstract}
The three-level neutral-point-clamped (3LNPC) converter is a promising multilevel topology in the application of mega-watts wind power generation system. However, the growing requirements by grid codes may impose high stress and even give reliability problem to this converter topology. This paper investigates the loss and thermal performances of a $10 \mathrm{MW} 3 \mathrm{~L}-\mathrm{NPC}$ wind power inverter undergoing Low Voltage Ride Through (LVRT) operation. A series of new space vector modulation methods are then proposed to relocate the thermal loading among the power switching devices. It is concluded that, with the proposed modulation methods, the thermal distribution in the 3L-NPC wind power inverter undergoing LVRT becomes more equal, and the junction temperature of the most stressed devices can be also relieved. Also the control ability of DC-bus neutral point potential, which is one of the crucial considerations for the 3L-NPC converter, is even more improved by the proposed modulation methods. ${ }^{1}$
\end{abstract}

\section{INTRODUCTION}

The European Union is committed to source $20 \%$ of its energy from renewables by 2020 [1]. As the most promising candidate, the wind energy production integrated into the power grid is booming all over the world. Meanwhile, the power capacity of a single wind turbine is increasing continuously to reduce the price pr. produced $\mathrm{kWh}$, as the cutting-edge achievement, $7 \mathrm{MW}$ offshore wind turbines have already been presented on the market [2]-[5]. Consequently, due to much more significant impacts to the power grid after a failure or disconnection than ever before, the wind power generation system is required to be more reliable and able to withstand grid disturbances. The Transmission System Operators (TSO) have issued stricter Low Voltage Ride Through (LVRT) grid codes [6], moreover, it is becoming a need that the wind power

Copyright (c) 2009 IEEE. Personal use of this material is permitted. However, permission to use this material for any other purposes must be obtained from the IEEE by sending a request to pubs-permissions@ieee.org.

$\mathrm{Ke} \mathrm{Ma}$ is now with the Department of Energy Technology, Aalborg University, Aalborg-9220, Denmark. (Tel: +45 21369447, e-mail: kema@et.aau.dk.)

Frede Blaabjerg is now with the Department of Energy Technology, Aalborg University, Aalborg-9220, Denmark. (e-mail: fbl@et.aau.dk). generation system should also provide reactive current (up to $100 \%$ rated current capacity of converter) to contribute to the grid recovery when LVRT is present [7]. A lot of work has been done related to the control of the wind power converter to satisfy the grid codes during LVRT [8], [9]. However, the loss and thermal performances under this condition, especially when using MW full-scale power converters, are important and interesting topics needed to be clarified.

It is expected that $10 \mathrm{MW}$ wind turbines with PMSG and full-scale power converter will be the next long-term target to be conquered according to the technology trends [2]-[5]. In most cases, the multi-level converter topologies will be demanded to handle such a high power with medium voltage ratings [10]-[17]. As the most commercialized multilevel converter [12]-[17], three-level neutral-point-clamped (3LNPC) topology seems to be a promising candidate for the 10 MW full-scale wind power converter [18]-[20], as shown in Fig. 1, where $T_{\text {out }}$ is the outer switch, $D_{\text {out }}$ is the outer freewheeling diode, $T_{\text {in }}$ is the inner switch, $D_{\text {in }}$ is the inner freewheeling diode, $\mathrm{D}_{\mathrm{npc}}$ is the clamping diode.

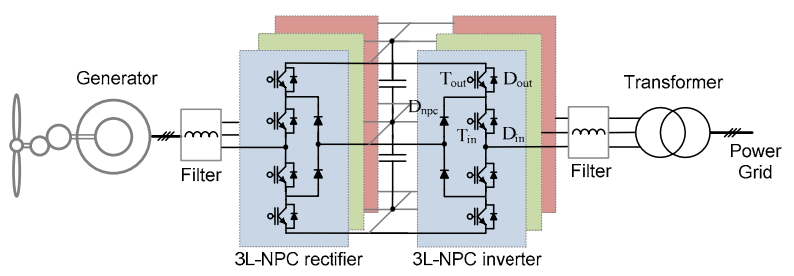

Fig. 1. Three-level neutral point clamped converter used in a wind turbine.

One drawback of 3L-NPC converter is the unequal loss distribution among the inner switches $T_{\text {in }}$ and $D_{\text {in }}$, the outer switches $\mathrm{T}_{\text {out }} / \mathrm{D}_{\text {out }}$ and the neutral point clamped (NPC) diodes $\mathrm{D}_{\mathrm{npc}}$, as indicated in Fig. 1. The inefficient and unequal utilization of power devices may limit the maximum achievable power, switching frequency and reliability of the whole converter system. Some works are proposed to improve this drawback with two-level modulation at lower modulation index [21], [22] or active neutral point clamped (NPC) switches [15]-[17]. But they have the disadvantages of either poorer control ability of neutral point potential or extra power switching devices. In [23] a modulation sequence is proposed to relieve the device thermal stress under LVRT, but it introduces extra switching events and the neutral point control method will compromise the thermal relieving effects. The problems of 3L-NPC converter may 
raise the uncertainty and restrict the availability of this converter topology in the application of wind turbines.

In this paper, the loss and thermal distribution among the power semiconductors of three-level neutral-point-clamped (3L-NPC) inverter are first investigated, the analysis is focusing on the application of $10 \mathrm{MW}$ wind turbines which is undergoing the low voltage ride through (LVRT) condition. Afterwards a series of new space-vectormodulation sequences which aim to reduce the stress of the "hottest" power devices and more equal thermal distribution are proposed. The neutral point (NP) potential control performances for the proposed modulation methods are also evaluated.

\section{THERMAL DISTRIBUTIONS OF 3L-NPC INVERTER UNDER LVRT}

A $10 \mathrm{MW}$ medium-voltage 3L-NPC wind power inverter is basically designed for a case study. As summarized in Table I, all of the power devices have the commutated voltage of $2.8 \mathrm{kV}$ in order to utilize the dominant $4.5 \mathrm{kV}$ high-power semiconductors available on the market, and the rated DC bus voltage can be determined at $5.6 \mathrm{kV}$. Normal continuous Space Vector Modulation (SVM) method for 3LNPC converter is applied and the carrier frequency is typically designed to be $800 \mathrm{~Hz}$ in order to get an acceptable switching loss in the power devices. The output filter inductance is designed to limit the maximum current ripple to $25 \%$ of the rated current amplitude, and the filter capacitance is not taken into account. For simplicity of analysis and keep the analysis focus on the power loss and thermal behavior of the power semiconductors, the power grid is considered as three ideal AC voltage sources, the DC bus capacitance is assumed high, and the transformers are assumed ideal.

The simulations are carried out based on PLECS Blockset in Simulink software [24], press-pack IGCT 5SHY 40L4511 from ABB (commutated voltage $2.8 \mathrm{kV} /$ maximum $3.6 \mathrm{kA}$, optimized for medium frequency switching) and freewheeling diodes 5SDF $10 \mathrm{H} 4503(2.8 \mathrm{kV} /$ maximum 4 $\mathrm{kA}$, recommended by the manufacturer) are chosen as the switching devices in this case study.

Table I. Parameters of a 10 MW 3L-NPC wind power inverter for case study (rated condition).

\begin{tabular}{|l|c|}
\hline Rated output active power $P_{o}$ & $10 \mathrm{MW}$ \\
\hline DC bus voltage $V_{d c}$ & $5.6 \mathrm{kV} \mathrm{DC}$ \\
\hline "Rated primary side voltage $V_{p}$ & $3.3 \mathrm{kV} \mathrm{rms}$ \\
\hline Rated line-to-line grid voltage $V_{g}$ & $20 \mathrm{kV} \mathrm{rms}$ \\
\hline Rated phase current $I_{\text {phase }}$ & $1750 \mathrm{~A} \mathrm{rms}$ \\
\hline Carrier frequency $f_{c}$ & $800 \mathrm{~Hz}$ \\
\hline Filter inductance $L_{f}$ & $1.13 \mathrm{mH}(0.2 \mathrm{p.u.})$. \\
\hline
\end{tabular}

* Line-to-line voltage in the primary windings of transformer.
As an extreme example, the inverter is set under the LVRT condition, when the grid voltage dips to 0.05 p.u. providing $100 \%$ rated reactive current to the power grid according to [7]. Because of the action of a DC bus chopper, the DC bus voltage during LVRT is assumed to be maintained at $10 \%$ higher than the rated value. The simulation output of the 3L-NPC inverter with normal Space Vector Modulation (SVM) sequence in two fundamental cycles is shown in Fig. 2. It can be seen that the output features of the converter during LVRT are quite different from the normal operation: the zero voltage level has longer dwelling time compared to the positive and negative voltage levels because of relative lower modulation index. The phase current lags 90 degree to the grid phase voltage because the inverter achieves $100 \%$ rated reactive current injection $(1.75$ $\mathrm{kA}$ rms) into the power grid.

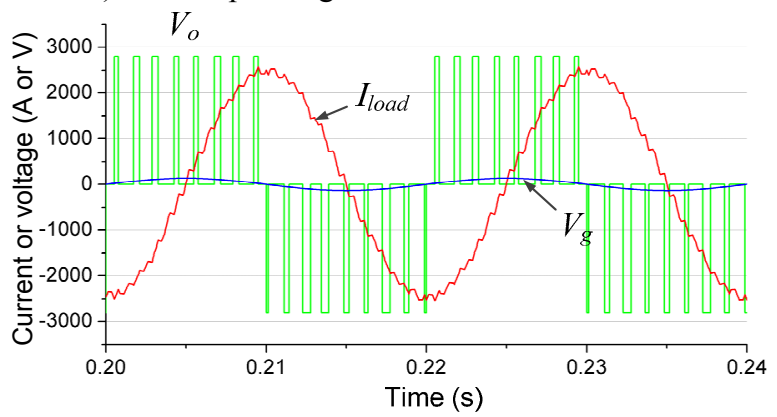

Fig. 2. Simulation outputs of 3L-NPC inverter with normal space vector modulation, ( $V_{o}$-output voltage pulses, $V_{g}$-grid voltage, $I_{\text {load }}{ }^{-}$ phase current. $V_{g}=0.05$ p.u., $100 \%$ rated reactive current).

When the operating condition for $3 \mathrm{~L}-\mathrm{NPC}$ wind power inverter is settled, the loss model can be applied based on the current and voltage information in the power devices. Afterwards the thermal model is applied to the loss dissipation, and junction temperature of each power device in 3L-NPC inverter can thereby be acquired. The loss model shares the same idea as in [24],[25], which is a commonly accepted method for the loss evaluation of power semiconductor devices. The thermal models of a single switch and clamping diode are indicated in Fig. 3 [26], [27], in which the thermal impedance from junction to case $Z_{(j-c)}$ is modeled as a four-layers Foster RC network, as shown in Fig. 4 , and it has to be transferred to the equivalent Cauer network for simulation [24]. Each of the thermal parameters can be found from the manufacturer datasheets and they are summarized in Table II, where the thermal resistance $\mathrm{R}_{\text {th }}$ will decide the steady state level of junction temperature, and the time constant $\tau$ (decided by $R_{t h}$ and thermal capacitance $C_{t h}$ ) will decide the dynamic performance of the junction temperature, their relationship is governed by [24]:

$$
\tau=R_{t h} \cdot C_{t h}
$$

It is noted that the Foster thermal network will be automatically transferred to the equivalent Cauer RC network in PLECS for the convenience of extending thermal impedance from case to ambient [24]. Due to the normally 
large thermal capacitance, the heat sink temperature is set to be $60{ }^{\circ} \mathrm{C}$ and considered constant during the converter operation. However it may be changed depending on the operation site.

The steady state mean junction temperature of each power device can be calculated as follows according to [26]:

$$
T_{j I G C T / \text { Diode }}=T_{H}+P_{I G C T / \text { Diode }} \cdot\left(R_{t h(j-c)}+R_{t h(c-h)}\right)
$$

$T_{\text {jIGCT/Diode }}$ and $P_{\text {IGCT/Diode }}$ represent the junction temperature and the total loss in a single IGCT or diode respectively, $T_{H}$ is the heat sink temperature, $R_{t h(j-c)}$ is the thermal resistance of IGCT or Diode from junction to case, $R_{t h(c-h)}$ is the thermal resistance of IGCT or Diode from case to heat sink.

The junction temperature distribution for each power device with relation to the grid voltage is shown in Fig. 5, in which the situations that the inverter needs to provide $100 \%$ rated reactive current are indicated. It can be seen that the

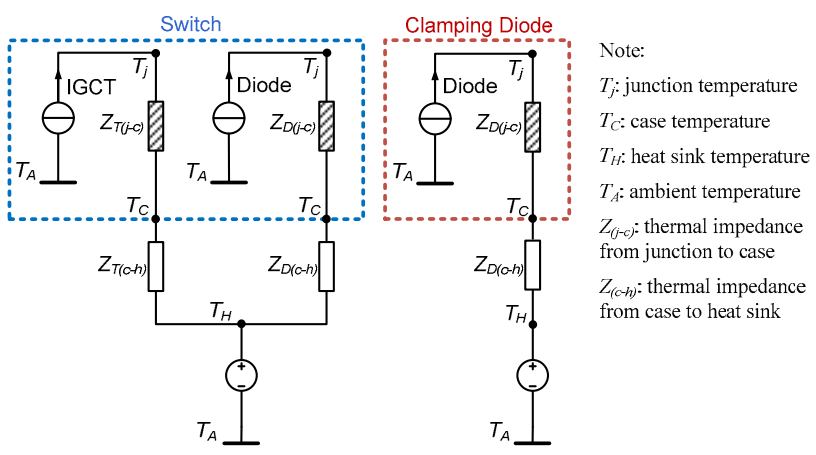

Fig. 3. Thermal models of the used power devices.

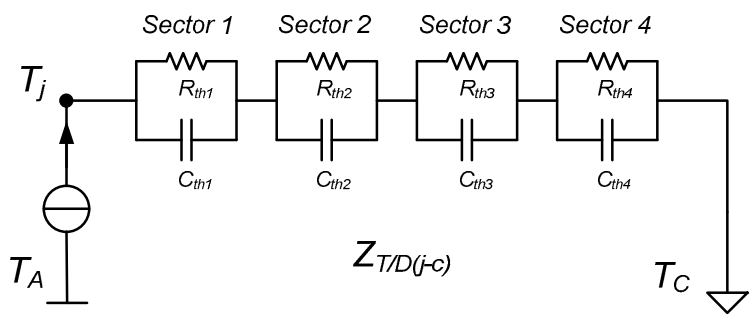

Fig. 4. Thermal model of the impedance $Z_{T(j-c)}$ or $Z_{D(j-c)}$ from junction to case in Fig. 3. (It has to be transferred to equivalent Cauer network for simulation).

Table II: Parameters of thermal impedance for IGCT/diode.

\begin{tabular}{|c|c|c|c|c|c|}
\hline \multirow{2}{*}{$\begin{array}{c}\text { Thermal } \\
\text { Impedance }\end{array}$} & \multicolumn{4}{|c|}{$\boldsymbol{Z}_{\text {TDD(j-c) }}$} & \multirow{2}{*}{$\boldsymbol{Z}_{\boldsymbol{T} \boldsymbol{D}(\mathrm{c}-\mathrm{h})}$} \\
\cline { 2 - 6 } & Sector 1 & Sector 2 & Sector 3 & Sector 4 & \\
\hline $\mathrm{R}_{\mathrm{iIGCT}}(\mathrm{K} / \mathrm{kW})$ & 5.562 & 1.527 & 0.868 & 0.545 & 3 \\
\hline$\tau_{\text {ilGCT }}(\mathrm{s})$ & 0.5119 & 0.896 & 0.0091 & 0.0024 & - \\
\hline $\mathrm{R}_{\mathrm{iDiode}}(\mathrm{K} / \mathrm{kW})$ & 7.705 & 2.748 & 1.009 & 0.539 & 3 \\
\hline$\tau_{\text {iDiode }}(\mathrm{s})$ & 0.5244 & 0.0633 & 0.0065 & 0.0015 & - \\
\hline
\end{tabular}

*Sector 1-4 means different layers of RC lump circuit in Fig. 4. thermal distribution of 3L-NPC inverter under LVRT is quite unequal and inefficient: as the "hottest" power devices, the NPC diodes $D_{\text {npc }}$ and inner switch $T_{\text {in }}$ has $15 \mathrm{~K}$ to $25 \mathrm{~K}$ higher junction temperatures than the average level of all power devices at around $80{ }^{\circ} \mathrm{C}$. While the most "cold" device $\mathrm{D}_{\text {in }}$ is barely loaded and has up to $40 \mathrm{~K}$ lower junction temperature than the "hottest" device $\mathrm{D}_{\mathrm{npc}}$. The extreme unequal thermal distribution happens when the grid voltage dips close to zero. It is obvious that the thermal optimization target for 3L-NPC wind power inverter under LVRT is to reduce the junction temperature in the NPC diodes $D_{n p c}$ and

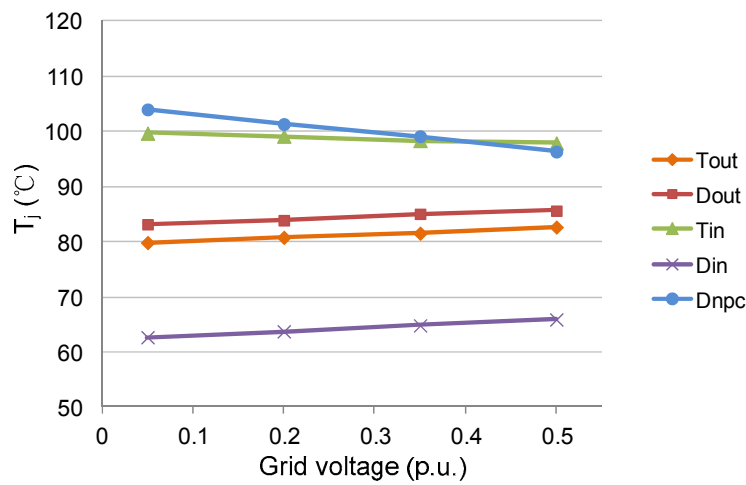

Fig. 5 Thermal distribution vs. grid voltage during Low Voltage Ride Through (100\% reactive power is needed).

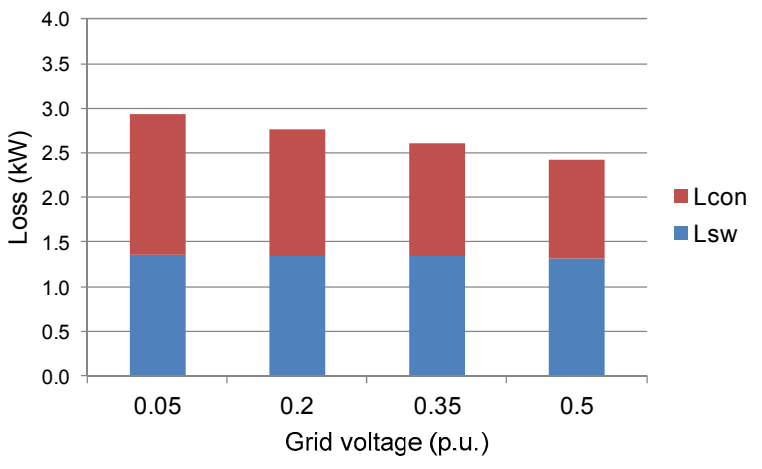

(a) Clamping diode Dnpc.

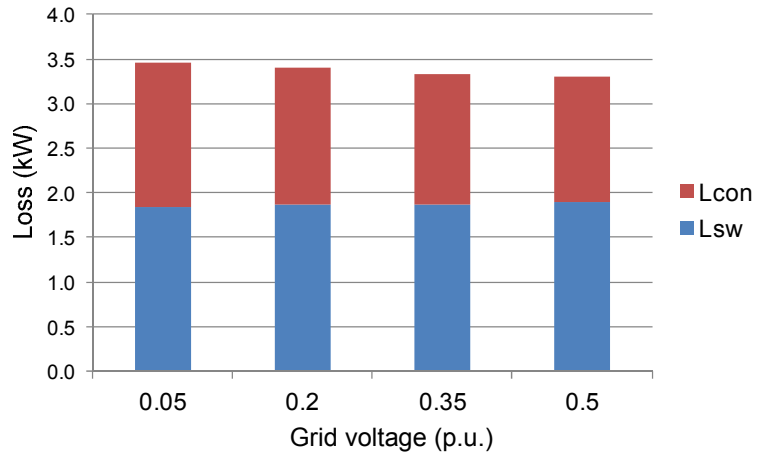

(b) Inner switch Tin.

Fig. 6. Loss distribution of the 3L-NPC wind power inverter under LVRT (Lcon conduction loss, Lsw switching loss). 
the inner switch $\mathrm{T}_{\mathrm{in}}$, while at the same time to achieve a more equal junction temperature distribution.

The loss distribution of the clamping diode $\mathrm{D}_{\mathrm{npc}}$ and inner switch $\mathrm{T}_{\text {in }}$ in $3 \mathrm{~L}-\mathrm{NPC}$ inverter when grid voltage dips to 0.05 , 0.2, 0.35 and 0.5 p.u. is shown in Fig. 6 (a) and Fig. 6 (b). It can be seen that the conduction loss in the NPC diode is slightly more dominant while the switching loss in $\mathrm{T}_{\text {in }}$ is more dominant. Referring to the switching process of the $3 \mathrm{~L}$ NPC converter [21], the thermal stress in $D_{\text {npc }}$ and $T_{\text {in }}$ will be effectively relieved by reducing the dwelling time of zero voltage level (reduced conduction loss) and reducing the commutations involving zero voltage level (reduced switching loss).

When implementing the space vector modulation for $3 \mathrm{~L}$ NPC inverter, the reference vector under extreme LVRT is mainly located in the inner hexagon of space vector diagram. In this special area, there are one or two redundancies for each of the switching state. The switching state redundancy enables quite a lot of possibilities to arrange the "threenearest" state vectors - it is possible that some of the state vectors sequence could modify the loss distribution and achieve the thermal optimization target for the 3L-NPC wind power inverter under LVRT.

\section{Generation Method for Space Vector MODULATION SEQUENCES}

For simplicity, the sequence generation method is demonstrated only based on sector I (0-60 degree) in the space vector diagram for $3 \mathrm{~L}-\mathrm{NPC}$ converter, and other sectors will share similar analysis. The detailed information of sector I is shown in Fig. 7, in which each of the state vectors group and sub-regions (A-D) are illustrated. As mentioned before, the voltage reference vector $V_{\text {ref }}$ in this sector mainly locates in region A under extreme LVRT condition. The reference can be synthesized by the three nearest state vectors: one from the redundant short vectors group 211/100 (red), one from the other redundant short vectors group 221/110 (blue) and one from the redundant zero vectors group 000/111/222 (black). The numbering " 2 ", " 1 ", " 0 " of the state vectors represent that a certain phase is connected by the converter to the positive DC bus, the neutral point and the negative DC bus respectively [21], [28]-[30].

It is interesting that when using all of the state vectors including the redundant ones in region A to synthesize the reference vector $\boldsymbol{V}_{r e f}$, the sequence arrangement is unique if the least switching process and symmetric pulses for each phase are realized, as shown in Fig. 8. All of the state vectors in region $\mathrm{A}$ are visited without unnecessary switching processes and abrupt voltage level changes: Output voltage level in each phase gradually change from negative to zero and then from zero to positive (reverse fashion is from positive to zero and then to negative). Other applicable modulation sequences can be generated by eliminating some redundant state vectors in the "complete" vector sequence.

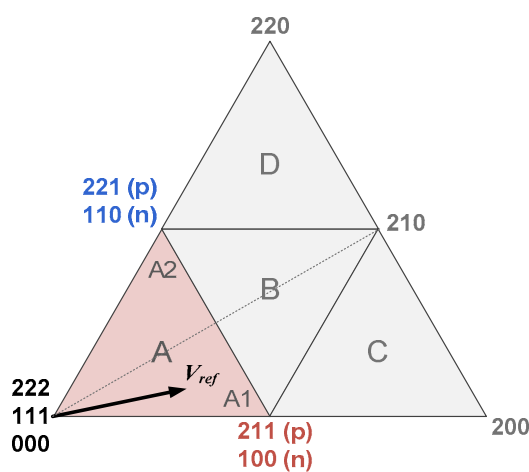

Fig. 7. Sector I of the space vector diagram for $3 \mathrm{~L}-\mathrm{NPC}$ converter.
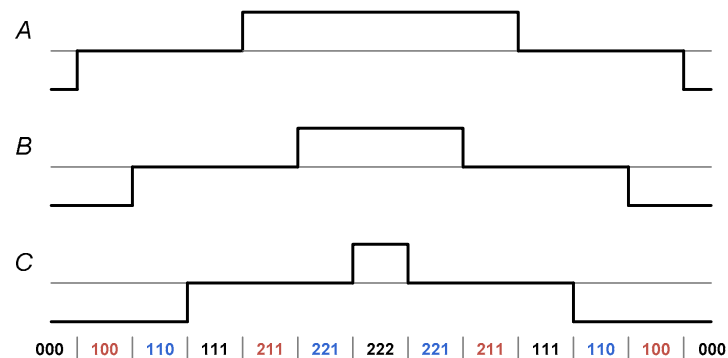

Fig. 8. The "complete" vector sequence in region A.

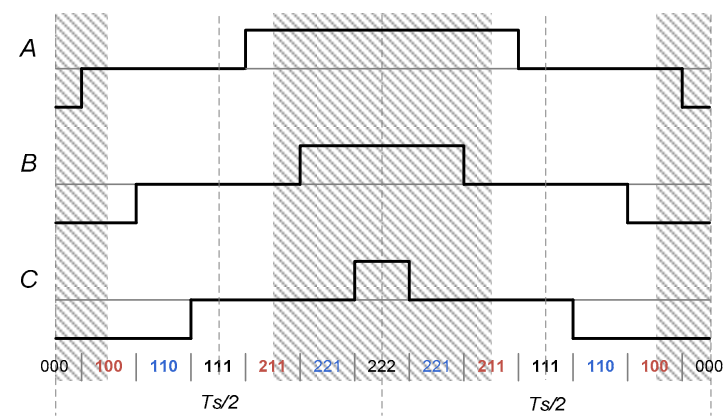

(a) Region A1 (0-30 degree)

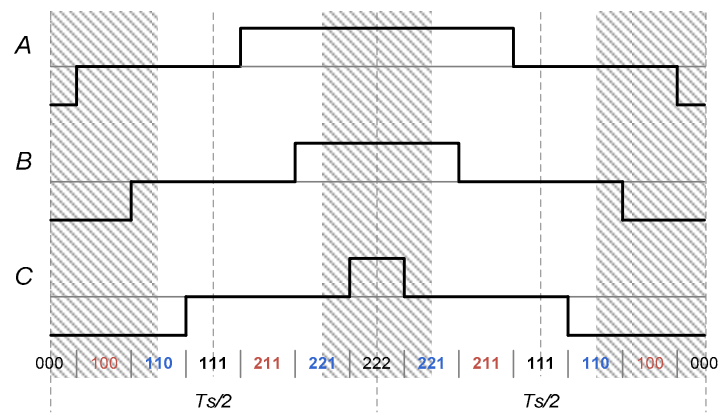

(b) Region A2 (30-60 degree)

Fig. 9. Vector sequences for normal space vector modulation, (grey parts mean eliminated state vectors, region A, sector I). 
However, the sequence generation method must follow two criteria:

I. At least one state vector from each of the "three nearest" redundant vectors group has to be used in order to synthesize the desired reference voltage.

II. The state vectors have to be arranged in reverse fashion over one carrier cycle in order to smoothly connect with the succeeded vector sequence without extra switching processes.

An example of this vector sequence generation method is demonstrated in Fig. 9, in which the most commonly used SVM sequence (normal) for 3L-NPC converters in the region A1 (0-30 degree) and region A2 (30-60 degree) of sector I are generated. It is noted that the grey blocks in Fig. 9 indicate the eliminated redundant state vectors, and the used state vectors are arranged within one switching cycle. It can be seen that the two sequence generation criteria are satisfied.

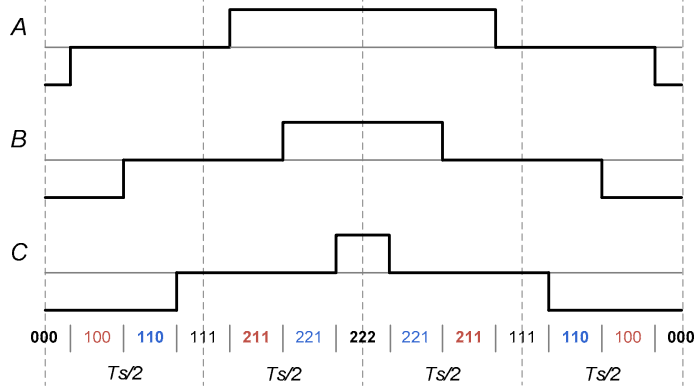

(a) O1 sequence arrangement

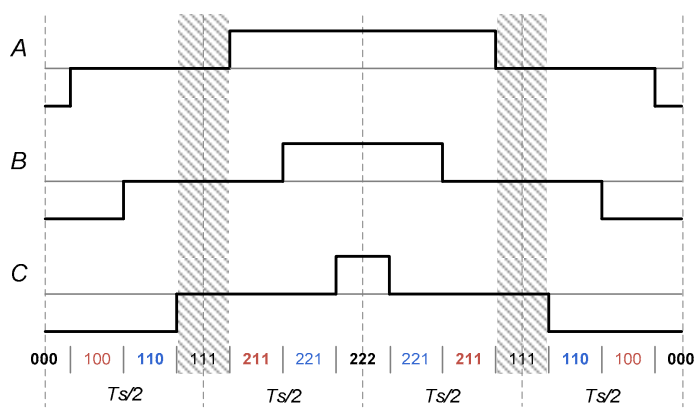

(c) $\mathrm{O} 2$ sequence arrangement

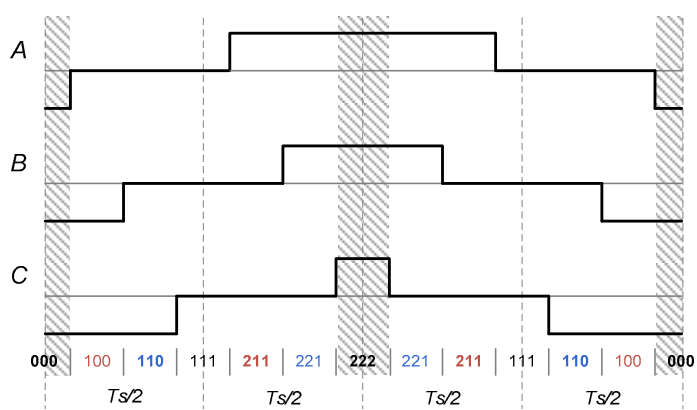

(e) $\mathrm{O} 3$ sequence arrangement

\section{THERMAL REDISTRIBUted MODULATIONS FOR 3L-NPC INVERTER UNDER LVRT}

\subsection{Basic principles}

As mentioned before, the thermal optimized modulation target is to reduce the dwelling time of zero voltage level or decrease the commutations involving zero voltage level. Based on the proposed generation method for modulation sequence, a series special modulation sequences are of interest:

In order to reduce the output time for zero voltage level of 3L-NPC inverter during LVRT, one effective way is to reduce the activating time for the zero state vector " 111 " (because vector 111 outputs zero voltage level for all of the three phases). Fig. 10 (a) indicates a special modulation sequence which allocates the "complete" vectors of Fig. 8 within two switching cycles, it is called Optimized sequence

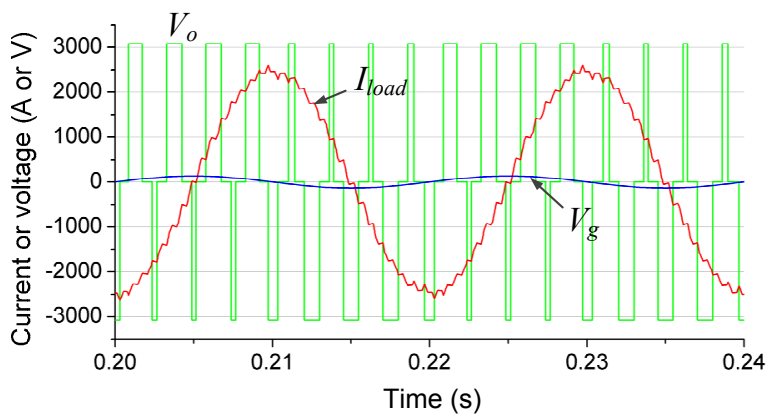

(b) Output of $\mathrm{O} 1$ sequence

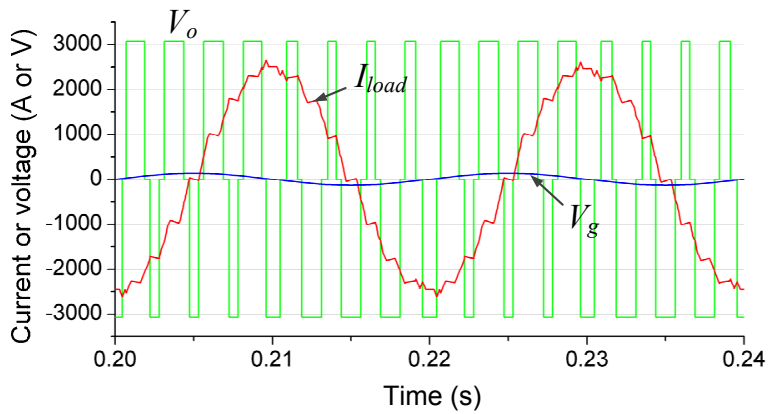

(d) Output of $\mathrm{O} 2$ sequence

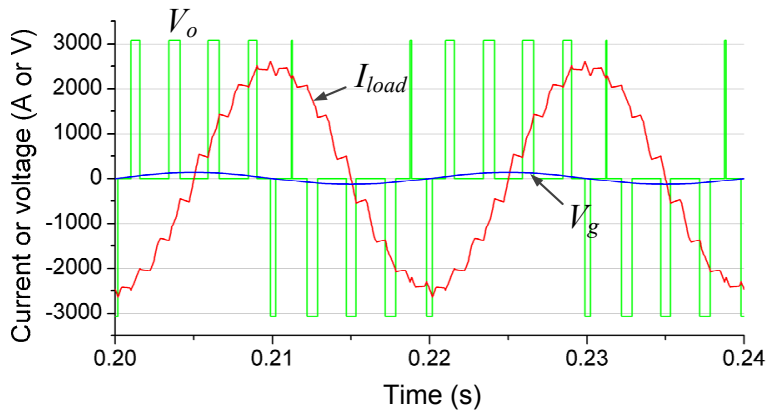

(f) Output of $\mathrm{O} 3$ sequence

Fig. 10. The loss and thermal redistributed modulation schemes. ( $V_{o}$-output voltage pulses, $V_{g}$-grid voltage, $I_{\text {load }}$-phase current. $V_{g}=0.05$ p.u., $100 \%$ rated reactive current). 


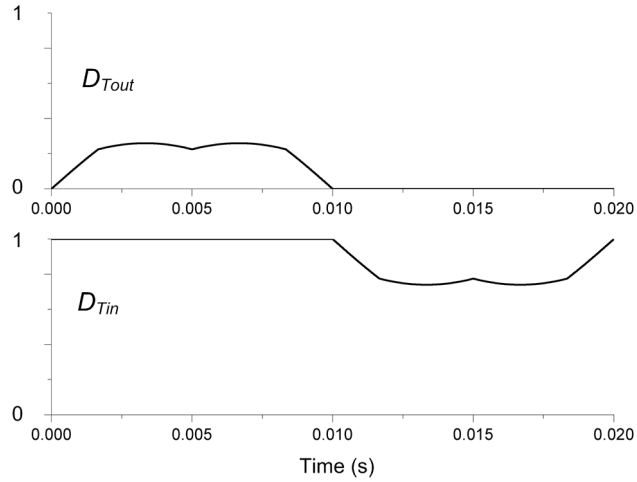

(a) Normal sequence

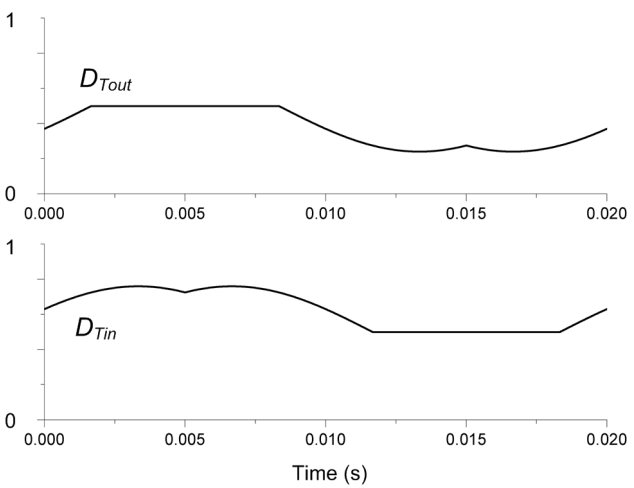

(c) $\mathrm{O} 2$ sequence

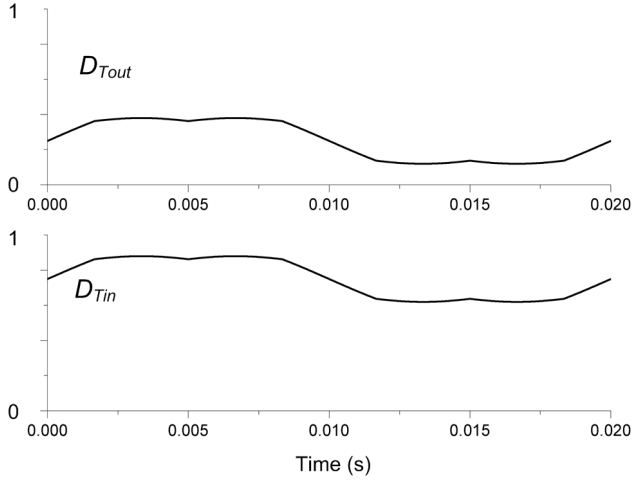

(b) $\mathrm{O} 1$ sequence

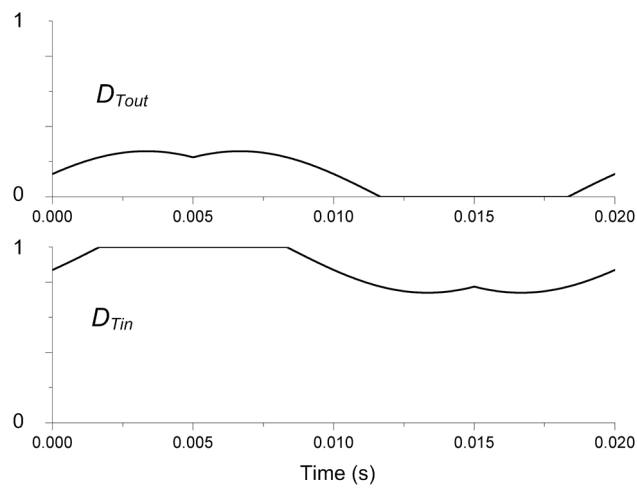

(d) $\mathrm{O} 3$ sequence

Fig. 11. SVM sequences in the form of carried-based modulation refences (duty ratio for the inner switch S1 and outer switch S2).

1 (O1) for convenience. In the normal SVM sequence shown in Fig. 9, the dwelling time for zero vector is all occupied by 111, while in the $\mathrm{O} 1$ sequence the zero vector is shared by 000,111 and 222, that means the output time for 111 in the $\mathrm{O} 1$ sequence is reduced and partly replaced by the equivalent vectors 000 and 222. The output waveforms of the $\mathrm{O} 1$ sequence are shown in Fig 10 (b), it can be seen that the output voltage of this modulation sequence alternates between positive, zero, negative voltage levels within neighboring switching cycles, which is quite different from the traditional three-level output pattern of 3L-NPC converter as indicated in Fig. 2. But the amplitude and phase angle of the load current is kept unchanged.

Another direct and basic idea is trying to avoid using the state vector "111" based on the "complete" sequence, as shown in Fig. 10 (c) which is called Optimized sequence 2 (O2) for convenience. It can be seen that the sequence generation criteria are satisfied and the zero state vectors 111 are totally replaced by the equivalent state vectors 000 and 222. The output waveforms of the $\mathrm{O} 2$ sequence are shown in Fig. 10 (d), in which the widths of both positive and negative output voltage pulses are expanded compared to the $\mathrm{O} 1$ sequence - that means further reduced dwelling time for zero voltage level.

In order to reduce the commutations involving zero voltage level, the third modulation sequence is generated in Fig. 10 (e), which eliminates the zero state vectors 000 and
222 and is called Optimized sequence 3 (O3) for convenience. It can be seen that for $\mathrm{O} 3$ sequence the commutations from zero to positive voltage levels in phase $\mathrm{C}$ and commutations from zero to negative voltage level in phase A are avoided in sector I (0-60 degree). The decreasing commutation times will benefit the switching loss reduction in the $3 \mathrm{~L}-\mathrm{NPC}$ inverter during LVRT. The output waveforms of the $\mathrm{O} 3$ sequence are shown in Fig. 10 (f), in which there are less output voltage pulses compared to the $\mathrm{O} 1$ and $\mathrm{O} 2$ sequence - that means reduced commutation times and less switching loss in $\mathrm{D}_{\mathrm{npc}}$ and $\mathrm{T}_{\mathrm{in}}$.

It is noticed that because there is redundancy for each of the state vector, all the used vectors in the proposed sequences are arranged within two switching cycles rather than one, as indicated at the bottom of Fig. 10 (a), Fig. 10 (c), Fig. 10 (e) respectively. As a result, compared to the normal sequence, the equivalent switching frequency in the proposed sequences is not further increased especially for $\mathrm{O} 1$ and $\mathrm{O} 2$.

For better understanding the proposed space vector modulation sequences in the form of carried-based modulation, they are transferred to the voltage references/duty ratio for the inner and outer switches of 3LNPC converter, as shown by $D_{\text {Tout }}$ and $D_{\text {Tin }}$ in Fig. 11. It can be seen that compared to the normal sequence in Fig. 11 (a), the $\mathrm{O} 1$ and $\mathrm{O} 2$ sequence generally move the level of voltage references, introducing larger duty ratio for the outer switch and smaller duty ratio for the inner switch, - this will lead to the longer dwelling time for the positive/negative voltage 
output and reduce the dwelling time of the zero voltage output. In respect to the $\mathrm{O} 3$ sequence, the duty ratio is very similar to the normal sequence but deviation can be found around the time for zero crossing of voltage reference - this means the dwelling time for zero voltage output is not significantly reduced. The $\mathrm{O} 3$ sequence is very similar to the modulation sequence proposed by [31], [32].

\subsection{Neutral point potential control and Total Harmonic Distortion}

In order to analyze the control ability of Neutral Point (NP) potential by the proposed modulation sequence, the neutral point current $i_{N P}$ which is the main reason for the DC bus unbalance will be focused. The used state vectors and their corresponding $i_{N P}$ for each of the modulation sequence are summarized in Table III. It can be seen that under the ideal condition, all of the proposed sequences will utilize the short vectors having zero accumulated $i_{N P}$ over a switching cycle. The difference is only for the zero vectors which have no impact to the NP current. Therefore the positive and negative DC bus can naturally be balanced in the proposed three sequences without any active methods.

In case of non-ideal situations, e.g. unbalanced load, the $i_{N P}$ will be disturbed in the 3L-NPC converter. It is well known that for the normal SVM sequence the average $i_{N P}$ can be controlled at zero by adjusting the time proportion of redundant vectors [33]-[35]. However according to Table III, in the normal sequence only one short vector (short 1) has redundancy and the other (short 2) doesn't, therefore the NP current control ability is limited by just half control freedom, It is also proven in [33] that the NP potential control can be only effective under certain power factors and modulation indexes. As for the proposed three sequences $\mathrm{O} 1, \mathrm{O} 2, \mathrm{O} 3$, both of the used short vectors have redundancies, which mean full control freedom for the NP current, therefore it is possible to achieve zero $i_{N P}$ within one switching cycle under all power factors and applicable modulation indexes. Consequently the NP potential control ability of all the proposed modulation sequences should be better than the normal SVM sequence.

Nevertheless, the proposed modulation sequences especially for $\mathrm{O} 2$ and $\mathrm{O} 3$ have no Total Harmonic Distortion (THD) improvements compared to the normal sequence. This can be also observed from the load current waveforms in Fig. 10 (d) and Fig. 10 (f), where the current distortion in the $\mathrm{O} 2, \mathrm{O} 3$ sequences are actually more than that in the normal sequence of Fig. 2. However it is noted that under the LVRT operation, which is an abnormal condition and normally last up to few seconds, the first control target is to make the grid converter survive when withstanding the grid voltage dips and providing large amount of reactive power support, therefore under the LVRT, THD is relatively less important performance for the wind power converter.

\subsection{Loss and thermal performances}

The loss distributions of the 3L-NPC wind power converter under LVRT when utilizing the normal and optimized vector sequences are compared in Fig. 12 (a), where $D_{c o n}$ and $T_{c o n}$ are the conduction loss in diode and IGCT respectively, $D_{s w}$ and $T_{s w}$ are the switching loss in diode and IGCT respectively. The comparison of steady state mean junction temperature distribution is shown in Fig. 12 (b), where the optimized sequence enabling $i_{N P}$ balance proposed in [23] is also indicated for comparison (called

Table III. The used vectors and neutral point current in sector I-III for different sequences within one switching cycle

\begin{tabular}{|c|c|c|c|c|c|c|c|c|c|}
\hline \multirow{2}{*}{ Sectors } & \multirow{2}{*}{$\begin{array}{l}\text { Vector } \\
\text { types }\end{array}$} & \multicolumn{2}{|c|}{01} & \multicolumn{2}{|c|}{ O2 } & \multicolumn{2}{|c|}{$\mathbf{O 3}$} & \multicolumn{2}{|c|}{ Normal $\left(0-30^{\circ}\right)$} \\
\hline & & Used vectors & $i_{N P}$ & Used vectors & $i_{N P}$ & $\begin{array}{c}\text { Used } \\
\text { vectors }\end{array}$ & $i_{N P}$ & $\begin{array}{c}\text { Used } \\
\text { vectors }\end{array}$ & $i_{N P}$ \\
\hline \multirow{5}{*}{ I } & \multirow{2}{*}{ Short 1} & 211 & $-i_{a}$ & 211 & $-i_{a}$ & 211 & $-i_{a}$ & 211 & $-i_{a}$ \\
\hline & & 100 & $i_{a}$ & 100 & $i_{a}$ & 100 & $i_{a}$ & 100 & $i_{a}$ \\
\hline & \multirow{2}{*}{ Short 2} & 110 & $-i_{c}$ & 110 & $-i_{c}$ & 110 & $-i_{c}$ & \multirow{2}{*}{110} & \multirow{2}{*}{$-i_{c}$} \\
\hline & & 221 & $i_{c}$ & 221 & $i_{c}$ & 221 & $i_{c}$ & & \\
\hline & Zero & 000111222 & 0 & 000222 & 0 & 111 & 0 & 111 & 0 \\
\hline \multirow{5}{*}{ II } & \multirow{2}{*}{ Short 1} & 121 & $-i_{b}$ & 121 & $-i_{b}$ & 121 & $-i_{b}$ & \multirow{2}{*}{121} & \multirow{2}{*}{$-i_{b}$} \\
\hline & & 010 & $i_{b}$ & 010 & $i_{b}$ & 010 & $i_{b}$ & & \\
\hline & \multirow{2}{*}{ Short 2} & 110 & $-i_{c}$ & 110 & $-i_{c}$ & 110 & $-i_{c}$ & 110 & $-i_{c}$ \\
\hline & & 221 & $i_{c}$ & 221 & $i_{c}$ & 221 & $i_{c}$ & 221 & $i_{c}$ \\
\hline & Zero & 000111222 & 0 & 000222 & 0 & 111 & 0 & 111 & 0 \\
\hline \multirow{5}{*}{ III } & \multirow{2}{*}{ Short 1} & 121 & $-i_{b}$ & 121 & $-i_{b}$ & 121 & $-i_{b}$ & 121 & $-i_{b}$ \\
\hline & & 010 & $i_{b}$ & 010 & $i_{b}$ & 010 & $i_{b}$ & 010 & $i_{b}$ \\
\hline & \multirow{2}{*}{ Short 2} & 011 & $-i_{a}$ & 011 & $-i_{a}$ & 011 & $-i_{a}$ & \multirow{2}{*}{011} & \multirow{2}{*}{$-i_{a}$} \\
\hline & & 122 & $i_{a}$ & 122 & $i_{a}$ & 122 & $i_{a}$ & & \\
\hline & Zero & $000 / 111 / 222$ & 0 & $000 / 222$ & 0 & 111 & 0 & 111 & 0 \\
\hline
\end{tabular}


A1A2 for simplicity). It can be seen that, the optimized sequences $\mathrm{O} 1, \mathrm{O} 2$ and $\mathrm{O} 3$ all achieve the loss and thermal redistribution among the power devices, the stress of $D_{n p c}$ and $T_{\text {in }}$ can be relieved under LVRT operation. The mean junction temperature reduction in the most stressed device $\mathrm{D}_{\text {npc }}$ and $\mathrm{T}_{\text {in }}$ are estimated to be up to $12 \mathrm{~K}$ and $8 \mathrm{~K}$ respectively when $\mathrm{O} 2$ modulation is applied. And $\mathrm{O} 3$ sequence shows another advantage in reducing the stress in $\mathrm{T}_{\text {out }}$ and $\mathrm{D}_{\text {out }}$ by $3 \mathrm{~K}$ and $5 \mathrm{~K}$ respectively. It is noted that the

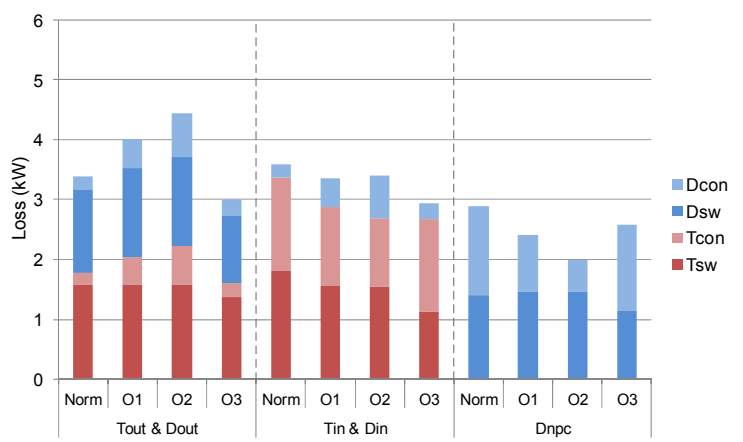

(a) Loss distribution. optimized sequence A1A2 in [23] has the similar thermal distribution as $\mathrm{O} 2$.

The dynamic thermal performance of $3 \mathrm{~L}-\mathrm{NPC}$ wind power inverter which goes from normal operation to extreme LVRT and then back to normal operation can also be simulated. The junction temperature of each power device when applying different modulation methods during LVRT is shown in Fig. 13. If the $\mathrm{O} 1$ and $\mathrm{O} 2$ modulation methods are applied, as shown in Fig. 13 (b) and Fig. 13 (c), the

Fig. 12. Thermal and loss comparison for the $3 \mathrm{~L}-\mathrm{NPC}$ wind power inverter under LVRT for different modulation sequences, $V_{g}=0.05$ p.u., $100 \%$ reactive power.

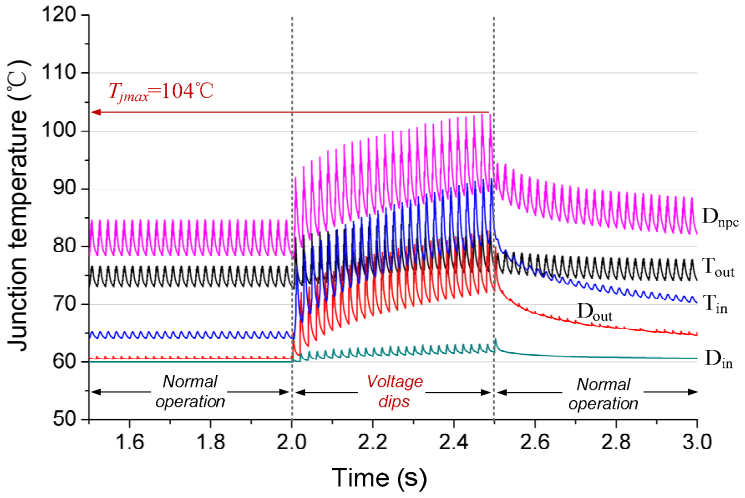

(a) Normal modulation during LVRT.

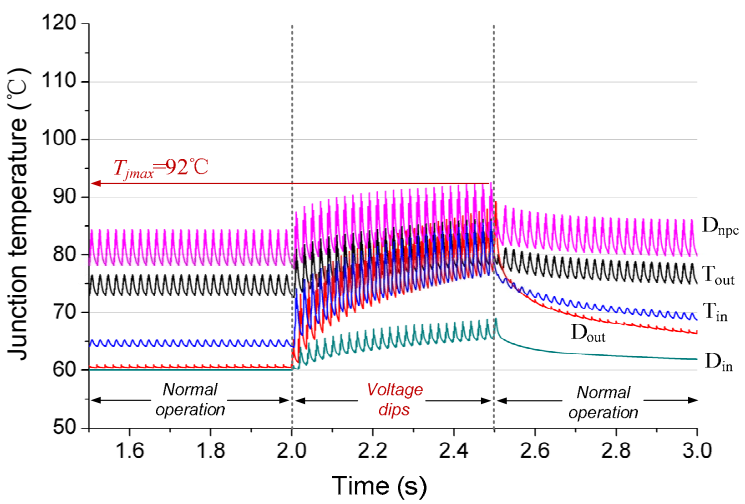

(c) $\mathrm{O} 2$ modulation during LVRT.

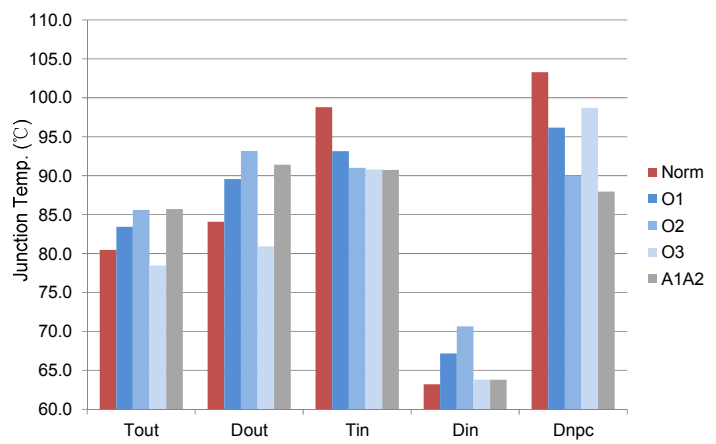

(b) Mean junction temperature comparison.

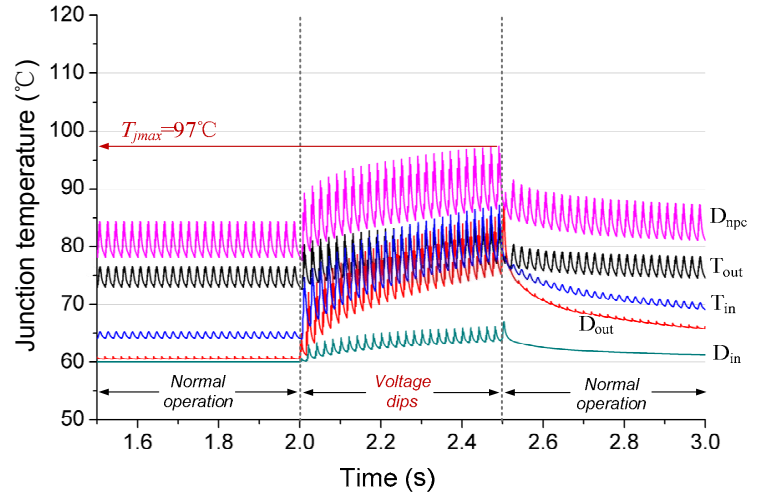

(b) O1 modulation during LVRT.

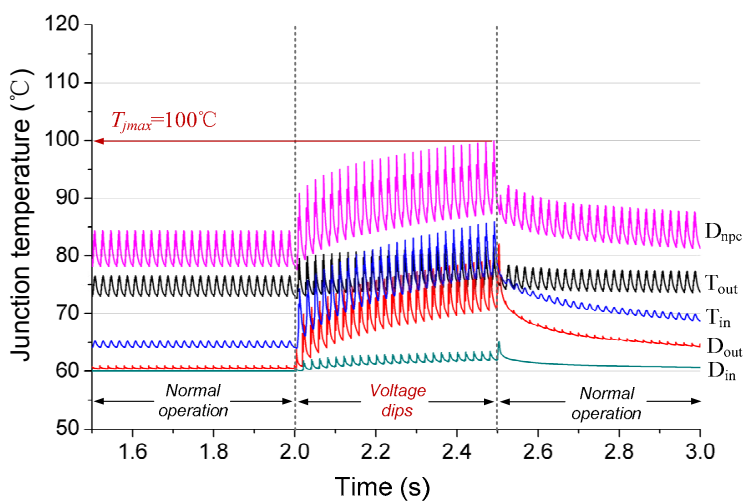

(d) O3 modulation during LVRT.

Fig. 13. Junction temperature dynamic response with a voltage dip time of $500 \mathrm{~ms}$ of different modulation sequences (from normal operation with wind speed $8 \mathrm{~m} / \mathrm{s}$ to 0.05 p.u. LVRT, and then back to normal operation). 
Table IV: Parameters for the experimental setup of 3L-NPC converter.

\begin{tabular}{|l|c|}
\hline DC bus voltage $V_{d c}$ & $200 \mathrm{~V}$ \\
\hline Modulation index $m$ & 0.3 \\
\hline Switching frequency $f_{s}$ & $2 \mathrm{kHz}$ \\
\hline Fundamental frequency $f_{o}$ & $50 \mathrm{~Hz}$ \\
\hline Load inductance $L_{\text {load }}$ & $28 \mathrm{mH}$ \\
\hline Load resistance $R_{\text {load }}$ & $1.2 \Omega$ \\
\hline Maximum load current $i_{\text {load }}$ & $3.5 \mathrm{~A}$ \\
\hline Phase angle $\theta$ & $80^{\circ}$ \\
\hline
\end{tabular}

maximum junction temperature in $\mathrm{D}_{\mathrm{npc}}$ and $\mathrm{T}_{\text {in }}$ are both reduced compared to the normal modulation in Fig. 13 (a). While the $\mathrm{O} 3$ modulation sequence shows more ability to reduce the junction temperature in $\mathrm{T}_{\text {out }}$ and $\mathrm{D}_{\text {out }}$ compared to $\mathrm{O} 1$ and $\mathrm{O} 2$ modulation methods.

The optimal sequence in respect to the thermal redistribution under LVRT depends on many factors like the voltage/power level, switching frequency, heat sink design and the used power switching devices [36]. Generally speaking $\mathrm{O} 2$ sequence shows better thermal performance where the conduction loss is dominant, like the application with high power and low switching frequency. While $\mathrm{O} 3$ sequence may show better thermal performance if the

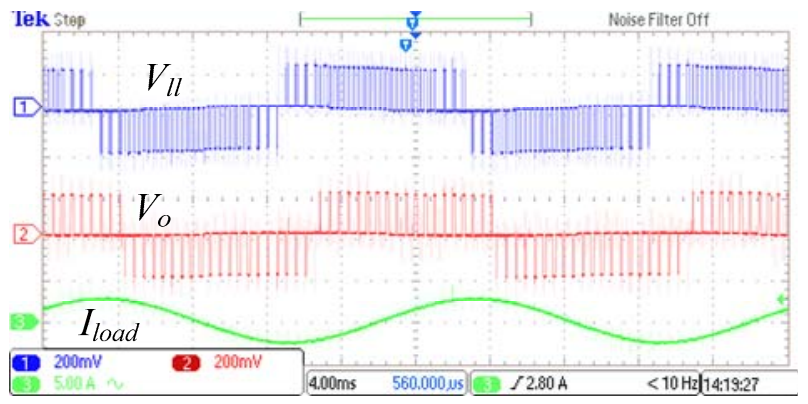

(a) Normal sequence

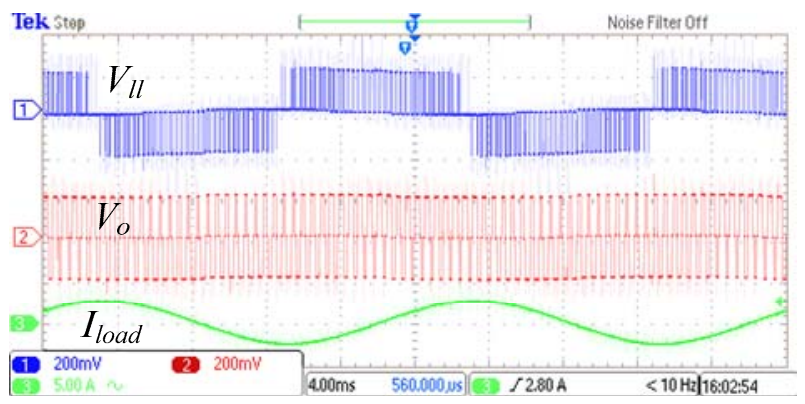

(c) $\mathrm{O} 2$ sequence switching loss is dominant like the application with low power and high switching frequency. However the preferred modulation sequence under LVRT should be evaluated case by case.

It is noted that, the proposed sequences are trying to move the loss from the most stressed devices to the less stressed ones. Although the total loss in the devices might not be significantly reduced, but the loss distribution is more equal, and the maximum junction temperature of the most devices can be reduced during LVRT. The more equal thermal distribution and maximum temperature limitation can be also observed from the dynamic thermal change in Fig. 13. The resulting maximum junction temperature reduction in the most stressed power devices may contribute to a life time extension of the converter according to e.g. the important Coffin-Masson life time model [37].

\section{EXPERIMENTAL RESULTS}

The proposed modulation sequences are validated on a downscale 3L-NPC converter, whose parameters are indicated in Table IV. It can be seen that a special $R L$ passive load is used to simulate the LVRT operating condition for the grid-connected converter with large amount of reactive power $\left(\theta=80^{\circ}\right)$ and low modulation index $(m=0.3)$.

Based on the setup the converter outputs with different modulation sequences are shown in Fig. 14, where the line to line voltage pulses (blue), phase voltage pulses (red), and load current (green) are indicated respectively. It can be seen that the different modulation sequences can achieve the

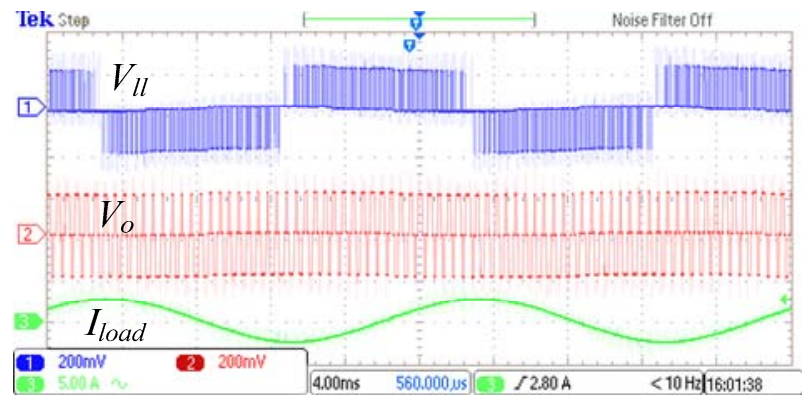

(b) O1 sequence

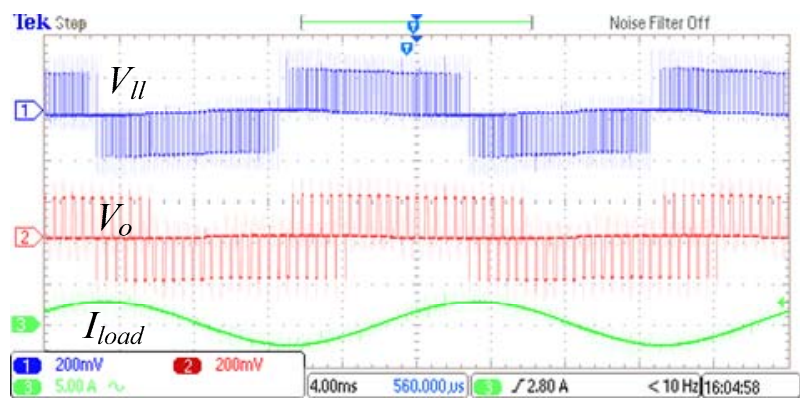

(d) $\mathrm{O} 3$ sequence

Fig. 14. Experimental outputs of different modulation sequences, $V_{l l}$-line to line voltage pulses (100V/div), $V_{o}$-phase voltage pulses $(100 \mathrm{~V} / \mathrm{div}), I_{\text {load }}$-load current ( $\left.5 \mathrm{~A} / \mathrm{div}\right)$, modulation index $M=0.3$ p.u., phase angle $\theta=80^{\circ}$. 

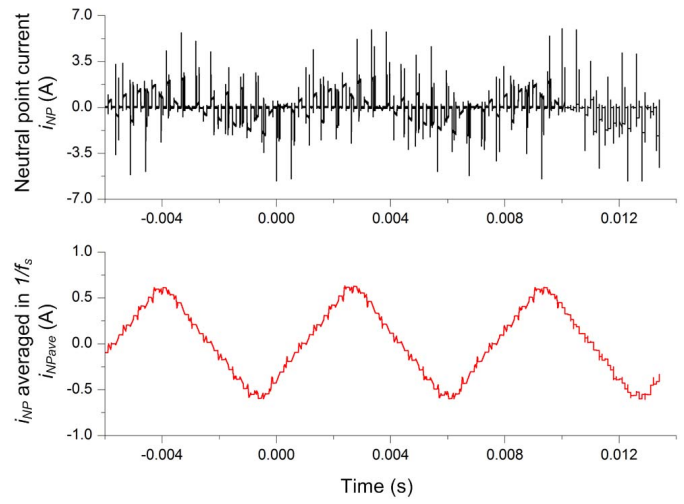

(a) Normal sequence
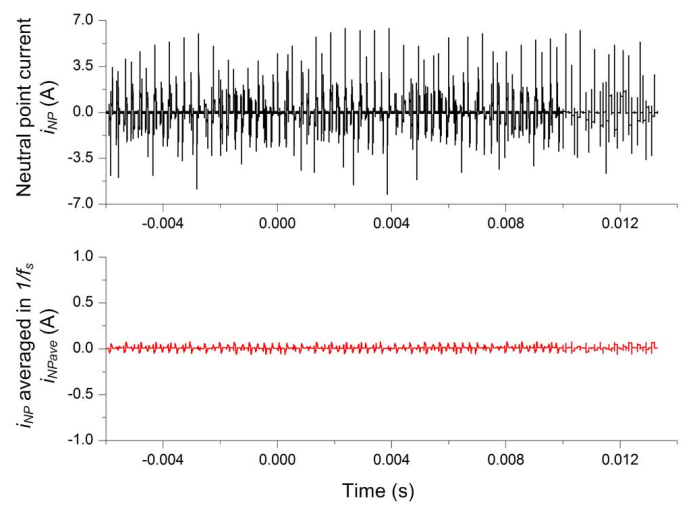

(c) $\mathrm{O} 2$ sequence
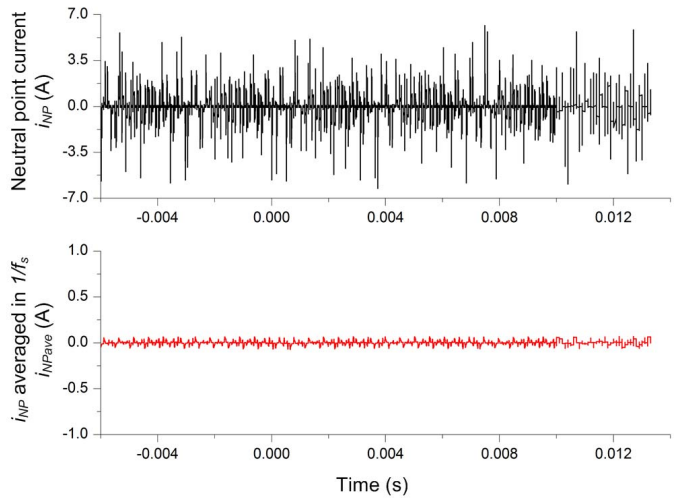

(b) $\mathrm{O} 1$ sequence
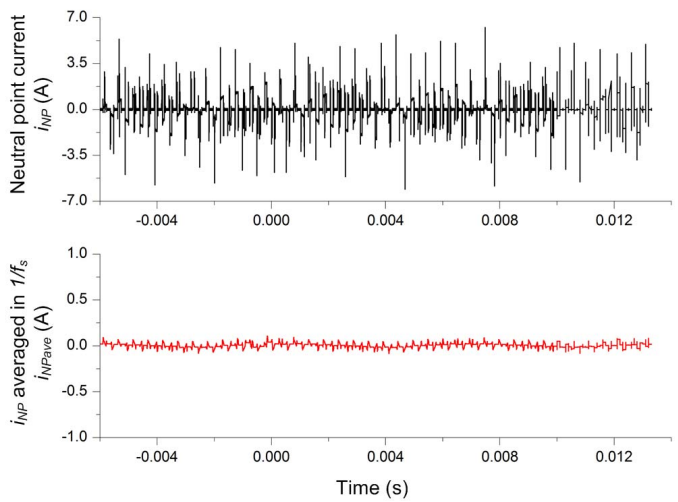

(d) $\mathrm{O} 3$ sequence

Fig. 15. Experimental neutral point current $i_{N P}$ and its switching-cycle-averaged value $i_{N P a v e}$ in different modulation sequences.

similar line-to-line voltage and load current, but the phase voltage are significantly different when various modulation sequences are applied, this agrees with the simulation results in Fig. 10.

Fig. 15 indicates the experimental NP current of the 3LNPC converter when different modulation methods are applied, it is noted that the active NP current control is not active. For clarity, both the instantaneous neutral point current $i_{N P}$ and its switching-cycle-averaged value $i_{N P a v e}$ are indicated. It can be seen that the $i_{\text {NPave }}$ in the proposed modulation sequences $\mathrm{O} 1-\mathrm{O} 3$ are almost eliminated, while the normal modulation sequence has acuminated-zero $i_{N P a v g}$ which oscillates at 0.2 p.u. amplitude and three times of the fundamental frequency.

\section{CONCLUSIONS}

According to the investigations in this paper, the thermal optimization target for 3L-NPC wind power inverter under extreme LVRT is to reduce the junction temperature in the NPC diode and inner switch, which are the hottest power devices of the whole inverter system.

By the proposed insight generation method for modulation sequence of $3 \mathrm{~L}-\mathrm{NPC}$ inverter, it is possible to develop a series of different thermal optimized modulation sequences. Compared to the normal modulation, the proposed thermal redistributed modulation sequences, which all enable full neutral point potential control ability, can effectively reduce the dwelling time or commutations involving zero voltage level output, achieving more equal thermal distribution and reliving the hottest power devices under extreme LVRT operation of 3L-NPC inverter. The proposed thermal optimized modulation methods are especially feasible during the LVRT operation, where the modulation index is relative low and more redundant switching states can be utilized.

It is noted that the thermal improvements by the proposed modulation methods during LVRT depend a lot on the design of heat sink system and used power switching devices, which may change the thermal distribution and the loading level in the most stressed devices, as analyzed in [36]. Therefore, the preferred modulation sequences in respect to the thermal redistribution under LVRT need to be evaluated case by case.

\section{References}

[1] European Commission Climate Action, "The EU climate and energy package", March 2007. 
[2] F. Blaabjerg, Z. Chen, S.B. Kjaer, "Power Electronics as Efficient Interface in Dispersed Power Generation Systems", IEEE Trans. on Power Electronics, 2004, vol. 19, no. 4, pp. 1184-1194.

[3] Z. Chen, J.M. Guerrero, F. Blaabjerg, "A Review of the State of the Art of Power Electronics for Wind Turbines," IEEE Trans. on Power Electronics, vol.24, no.8, pp.1859-1875, Aug. 2009.

[4] Website of Vestas Wind Power, Wind turbines overview, April 2011. (Available: http://www.vestas.com/).

[5] F. Blaabjerg, M. Liserre, K. Ma, "Power Electronics Converters for Wind Turbine Systems," IEEE Trans. on Industry Applications, vol 48, No. 2, pp.708-719, 2012.

[6] M. Altin, O. Goksu, R. Teodorescu, P. Rodriguez, B. BakJensen, L. Helle, "Overview of recent grid codes for wind power integration," Proc. of OPTIM'2010, pp.1152-1160, 2010.

[7] E.ON-Netz - Grid Code. High and extra high voltage, April 2006.

[8] S.M.Muyeen, R.Takahashi, T.Murata, J.Tamura, "A Variable Speed Wind Turbine Control Strategy to Meet Wind Farm Grid Code Requirements," IEEE Trans. on Power Systems, 2010, vol. 25, no. 1, pp. 331-340.

[9] A.Teninge, D. Roye, S.Bacha, "Reactive power control for variable speed wind turbines to low voltage ride through grid code compliance," Proc. of ICEM’2010, pp. 331-340, 2010.

[10] M. Liserre, R. Cardenas, M. Molinas, J. Rodriguez, "Overview of Multi-MW wind turbines and wind parks", IEEE Trans. on Industrial Electronics, Vol. 58, No. 4, pp. 1081-1095, April 2011.

[11] Hui Zhang, L.M. Tolbert, "Efficiency Impact of Silicon Carbide Power Electronics for Modern Wind Turbine Full Scale Frequency Converter," IEEE Trans. on Industrial Electronics, vol. 58, no. 1, pp. 21-28, Jan 2011.

[12] S. Kouro, M. Malinowski, K. Gopakumar, J. Pou, L. G. Franquelo, B. Wu, J. Rodriguez, M. A. Perez, J. I. Leon, "Recent Advances and Industrial Applications of Multilevel Converters," IEEE Trans. on Industrial Electronics, vol. 57, no. 8 , pp. $2553-2580,2010$

[13] D. Krug, S. Bernet, S. S. Fazel, K. Jalili, M. Malinowski, "Comparison of 2.3-kV Medium-Voltage Multilevel Converters for Industrial Medium-Voltage Drives," IEEE Trans. on Industrial Electronics, vol. 54, no. 6, pp. 2979-2992, 2007

[14] J. Rodriguez, S. Bernet, P. K. Steimer, I. E. Lizama, "A Survey on Neutral-Point-Clamped Inverters," IEEE Trans. on Industrial Electronics, vol. 57, no. 7, pp. 2219-2230, 2010.

[15] T. Bruckner, S. Bernet, H. Guldner, "The active NPC converter and its loss-balancing control," IEEE Trans. on Industrial Electronics, vol. 52, no. 3, pp.855-868, 2005.

[16] O. Apeldoorn, B. Odegard, P. Steimer, S. Bernet, "A 16 MVA ANPC-PEBB with 6 kA IGCTs," in Proc. of IAS'05, vol.2, pp. 818-824, 2005.

[17] O. S. Senturk, L. Helle, S. Munk-Nielsen, P. Rodriguez, R. Teodorescu, "Medium voltage three-level converters for the grid connection of a multi-MW wind turbine," in Proc. of EPE'09, pp: 1- 8, 2009.

[18] M. Malinowski, S. Stynski, W. Kolomyjski, M.P. Kazmierkowski, "Control of Three-Level PWM Converter Applied to Variable-Speed-Type Turbines," IEEE Trans. on Industry Applications, vol.56, no.1, pp.69-77, 2009.

[19] A. Faulstich, J.K. Stinke, F. Wittwer, "Medium voltage converter for permanent magnet wind power generators up to 5 MW," in Proc. of EPE'05, pp: 1- 9, 2005.

[20] S. Alepuz, S. Busquets-Monge, J. Bordonau, J. Gago, D. Gonzalez, J. Balcells, "Interfacing Renewable Energy Sources to the Utility Grid Using a Three-Level Inverter," IEEE Trans. on Industrial Electronics, vol. 53, no. 5, pp. 1504-1511, 2006.

[21] D. G. Holmes and T. A. Lipo, Pulse Width Modulation for Power Converters. New York: IEEE Press/Wiley-Interscience, 2003.

[22] T.Bruckner, D.G.Holmes, "Optimal pulse-width modulation for three-level inverters," IEEE Trans. on Power Electronics, vol. 20 , no. 1 , pp. 82-89, 2005.

[23] K. Ma, F. Blaabjerg, "Thermal Optimized Modulation Methods of Three-level Neutral-Point-Clamped Inverter for 10 MW Wind Turbines under Low Voltage Ride Through," IET Power Electronics, Vol. 5, No. 6, pp.920-927, 2012.

[24] User manual of PLECS blockset version 3.1, March 2011. (Available: http://www.plexim.com/files/plecsmanual.pdf).

[25] F. Blaabjerg, U. Jaeger, S. Munk-Nielsen and J. Pedersen, "Power Losses in PWM-VSI Inverter Using NPT or PT IGBT Devices," IEEE Trans. on Power Electronics, vol. 10, no. 3, pp. 358-367, May 1995.

[26] ABB Application Note: Applying IGBTs, May 2007.

[27] W. Lixiang, J. McGuire,R.A. Lukaszewski, "Analysis of PWM Frequency Control to Improve the Lifetime of PWM Inverter," IEEE Trans. on Industrial Electronics, vol. 47, no. 2, pp. 922929, 2011.

[28] J.Holtz, N.Oikonomou, "Neutral Point Potential Balancing Algorithm at Low Modulation Index for Three-Level Inverter Medium-Voltage Drives," IEEE Trans. on Industry Applications, vol.43, no.3, pp.761-768, 2007.

[29] Fei Wang, "Sine-triangle versus space-vector modulation for three-level PWM voltage-source inverters," IEEE Trans. on Industry Applications, vol.38, no.2, pp.500-506, 2002.

[30] B.P. McGrath, D.G. Holmes, T. Lipo, "Optimized space vector switching sequences for multilevel inverters," IEEE Trans. on Power Electronics, vol. 18, no. 6, pp. 1293-1301, 2003.

[31] S. Busquets-Monge, J. Bordonau, D. Boroyevich, S. Somavilla, "The nearest three virtual space vector PWM - a modulation for the comprehensive neutral-point balancing in the three-level NPC inverter," IEEE Power Electron. Letter, vol. 2, no. 1, pp. 11-15, Mar. 2004.

[32] S. Busquets-Monge, J. Bordonau, J. A. Beristain, "Comparison of losses and thermal performance of a three-level three-phase neutral-point-clamped dc-ac converter under a conventional NTV and the NTV2 modulation strategies," Proc. IECON" 2006, pp. 4819-4824, 2006.

[33] N. Celanovic, D. Boroyevich, "A comprehensive study of neutral-point voltage balancing problem in three-level neutral- 
point-clamped voltage source PWM inverters," IEEE Trans. on Power Electronics, 2000, vol. 15, no. 2, pp. 242-249.

[34] S. Srikanthan, M.K. Mishra, "DC Capacitor Voltage Equalization in Neutral Clamped Inverters for DSTATCOM Application ," IEEE Trans. on Industrial Electronics, vol. 57, no. 8, pp., August 2010.

[35] J. Zaragoza, J. Pou, S. Ceballos, E. Robles, C. Jaen, M. Corbalan, "Voltage-Balance Compensator for a Carrier-Based Modulation in the Neutral-Point-Clamped Converter," IEEE Trans. on Industrial Electronics, vol. 56, no. 2, pp. 305-314, Feb 2009.

[36] Ke Ma, Frede Blaabjerg, "The Impact of Power Switching Devices on the Thermal Performance of a $10 \mathrm{MW}$ Wind Power NPC Converter," Energies 5, no. 7: 2559-2577.

[37] I.F. Kovačević, U. Drofenik, J.W. Kolar, "New physical model for lifetime estimation of power modules," in Proc.of IPEC'10, pp. 2106-2114, 2010.

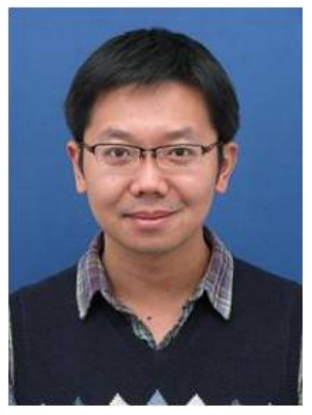

Ke Ma (S'09-M'11) He received the B.Sc. and M.Sc. degrees in electrical engineering from the Zhejiang University, Hangzhou, China in 2007 and 2010 respectively. He is currently working toward the Ph.D. degree in the Department of Energy Technology, Aalborg University, Denmark.

His research interests are in the multilevel power electronics converters and their application in the wind power generation system. He has received the IEEE Industry Applications Society IPCC third prize paper award in 2012 and a prize paper award at ISIE Poland 2011.

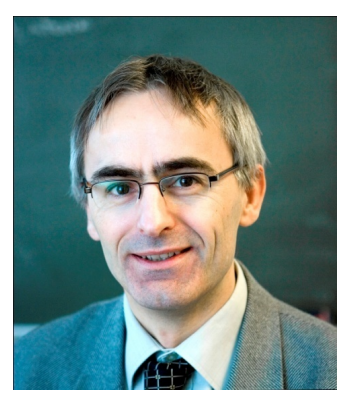

Frede Blaabjerg (S'86-M'88SM'97-F'03) He was employed at ABB-Scandia, Randers, from 19871988. During 1988-1992 he was PhD. student at Aalborg University, Denmark, became Assistant Professor in 1992, Associate Professor in 1996 and full professor in power electronics and drives in 1998. He has been part-time research leader at Research Center Risoe in wind turbines. In 2006-2010 he was dean of the faculty of Engineering, Science and Medicine and became visiting professor at Zhejiang University, China in 2009. His research areas are in power electronics and its applications like in wind turbines, PV systems and adjustable speed drives. He was the Editor-in-Chief of the IEEE Transactions on Power Electronics 2006-2012. He was Distinguished lecturer for the IEEE Power Electronics Society 2005-2007 and for IEEE Industry Applications Society from 2010-2011. He has been Chairman of of EPE'2007 and PEDG'2012 - both held in Aalborg.

$\mathrm{He}$ received the 1995 Angelos Award for his contribution in modulation technique and the Annual Teacher prize at Aalborg University. In 1998 he received the Outstanding Young Power Electronics Engineer Award from the IEEE Power Electronics
Society. He has received thirteen IEEE Prize paper awards and another prize paper award at PELINCEC Poland 2005. He received the IEEE PELS Distinguished Service Award in 2009 and the EPEPEMC 2010 Council award. Finally he has received a number of major research awards in Denmark. 